Artículo

\title{
Actualización de la cartografía edafológica del Estado de México: una herramienta para la planeación
}

\author{
Erasto Domingo Sotelo Ruiz ${ }^{1 \S}$ \\ Gustavo Cruz Bello ${ }^{2}$ \\ Antonio González Hernández ${ }^{3}$ \\ Román Flores López ${ }^{1}$
}

${ }^{1}$ Campo Experimental Metepec-INIFAP. Vialidad Adolfo López Mateos, carretera Toluca-Zitácuaro km 4.5, San José Barbabosa, Zinacantepec, Estado de México. CP. 51350. (flores.roman@inifap.gob.mx). ${ }^{2}$ Laboratorio de análisis socio territorial-Universidad Autónoma Metropolitana Cuajimalpa. Av. Vasco de Quiroga 4871, Santa Fe, Cuajimalpa, Ciudad de México. CP. 05348. (gcruzbel07@ gmail.com). ${ }^{3}$ Centro Nacional de Investigaciones Disciplinarias en Conservación y Mejoramiento de Ecosistemas ForestalesINIFAP. Av. Progreso No. 5, Barrio de Santa Catarina, Coyoacán, Ciudad de México. CP. 04010. (aglez6419@gmail.com).

${ }^{\S}$ Autor para correspondencia: soteloe@colpos.mx.

\section{Resumen}

El suelo es un cuerpo natural muy importante para el desarrollo de cultivos y especies vegetales, debido a que provee el soporte a las raíces de las plantas, además aporta nutrientes para su desarrollo y producción, dependiendo del tipo de suelo y sus propiedades. Conocer y cuantificar los suelos es necesario para planear las actividades agrícolas, pecuarias, forestales, urbanas, mineras y de conservación. Los objetivos del presente trabajo fueron: 1) actualizar la cartografía edafológica 1:50 000 por municipio del Estado de México; y 2) cuantificar la superficie de los suelos y conocer su ubicación para identificar áreas de reconversión productiva. La cartografía se generó con la digitalización de las cartas edafológicas escala 1:50 000, con un sistema de información geográfica (SIG), se generó su base de datos: suelos primarios, suelos secundarios, fase física, fase química y textura; esta base de datos se actualizó a la versión de suelos 2015 de la WRB. Los suelos con mayor superficie y más productivos son: Andosoles con 479908 ha, Feozems con 472718 ha, Vertisoles con 241485 ha y Cambisoles con 196047 ha. Estos predominan en los municipios de Aculco, Toluca, Acambay, Jilotepec, Axapusco, Ixtlahuaca y Almoloya de Juárez.

Palabras clave: actualización, cartografía digital, municipio, suelos.

Recibido: octubre de 2020

Aceptado: diciembre de 2020 


\section{Introducción}

La palabra 'suelo' tiene varios significados y se deriva de la palabra solum del latín que significa suelo. En su significado tradicional, el suelo es el medio natural para el desarrollo de las plantas terrestres, ya sea que tenga o no horizontes discernibles (Soil Survey Staff, 2014; IUSS Working Group WRB, 2015).

Los suelos, de manera natural, tienen cinco funciones principales: 1) soportar el crecimiento de las plantas superiores, principalmente por proveer un medio para las raíces y suministro de nutrimentos que son esenciales para todas las plantas; 2) controlar el destino del agua en el sistema hidrológico; 3) como sistema de reciclaje; 4) proveer un hábitat para un sinnúmero de organismos vivos como pequeños mamíferos, reptiles, insectos diminutos y diversidad de células microscópicas; y 5) el suelo juega un importante rol como medio ingenieril (Brady y Weil, 1999; Porta et al., 2003; Soil Survey Staff, 2014).

Los sistemas taxonómicos modernos de suelos y los más utilizados son la taxonomía de suelos (TS) y la base referencial mundial del recurso suelo (WRB), los cuales clasifican los suelos usando horizontes, propiedades y materiales de diagnóstico (Bockheim y Gennadiyev, 2000; Spaargaren, 2000; Wilding, 2000; IUSS Grupo de Trabajo WRB, 2007; Soil Survey Staff, 2014; IUSS Working Group WRB, 2015).

La TS consta de seis categorías: orden, suborden, gran grupo, subgrupo, familia y serie. La primera versión se publicó en 1960, la cual constaba de 10 órdenes. La versión de 1999 se modifica y consta 12 órdenes, donde se incluyen los Andosoles y los Gélisoles, los cuáles se conservan hasta la versión de 2014 (Soil Survey Staff, 1960; Soil Survey Staff, 1998; Soil Survey Staff, 2014). La clasificación de la WRB consta de dos categorías: la unidad y la subunidad. Ésta empezó su publicación en 1970 con 26 unidades y 104 subunidades, la versión de 1988 con 28 unidades y 153 subunidades, la de 1998 con 30 unidades y 533 subunidades, la versión de 2006 y 2015 constan de 32 unidades y las subunidades pueden ser todas las posibles combinaciones (FAO-UNESCO, 1970; FAO, 1988; FAO-ISRIC y SICS, 1998; IUSS Working Group WRB, 2006; IUSS Working Group WRB, 2015).

Los estudios de suelos surgen en Estados Unidos de América a principios de 1820 y toman como base a la escuela rusa, donde se parte de un concepto y una base geológica, la cual con el tiempo cambia a un concepto pedológico de suelos (Brevik y Hartemink, 2013). Los investigadores que realizaron estudios y mapearon los suelos tenían una formación con base geológica (Brevik, 2009; Brevik, 2010), por lo tanto, estos primeros mapas fueron esencialmente mapas de la geología superficial (Brevik y Hartemink, 2010).

El mapeo digital presenta muchas ventajas para mejorar, automatizar y actualizar los estudios de suelos, tales como: a) mapeo consistente; b) actualización rápida de los levantamientos de suelos; c) reducción de costos y tiempo; d) continuidad del conocimiento; y e) productos digitales (Zhu et al., 2001; McBratney et al., 2003; Hengl y Rossieter, 2003; Behrens y Scholten, 2006; Kozlova y Konyushkova, 2009). 
En la actualidad, los estudios para generar la cartografía de suelos se basan en predicciones y aplicación de modelos con base en propiedades. Esto se debe a lo tardado y costoso de la aplicación de las metodologías de levantamientos de suelos para la elaboración de cartografía edafológica. Entre estos estudios destacan los de Behrens et al. (2010a; 2010b) quienes proponen generar mapas de suelos basados en la elevación del terreno. Brevik y Hartemink (2013) mencionan que los mapas de suelos generados con estudios de suelos son importantes, porque proporcionan información valiosa de los mapas y del tiempo en que fueron elaborados.

Rosas et al. (2015) concluyen que el uso de más variables ambientales resulta en un incremento en la exactitud en modelos de predicción de mapas de suelos. Por su parte, Jafari et al. (2014) aplican modelos de predicción para el mapeo digital de los grandes grupos de suelos. Finalmente, Shi et al. (2009) mencionan que el conocimiento de los suelos derivado de los estudios realizados por los científicos de la ciencia del suelo es una guía para el desarrollo y planeación de las actividades agropecuarias, concluyen que el mapeo digital de suelos es importante para la generación y cartografía de mapas de suelos.

Para el caso de México, Ortiz et al. (1994) hacen una adecuación de la versión de la FAO de 1988 a los suelos de México, donde realizan los cambios de las unidades que desaparecen de los mapas de suelos del país (FAO, 1988). Además, la SEMARNAP (1996) estableció los fundamentos y adecuaciones de las unidades de suelos. Por su parte, el Instituto Nacional de Estadística Geografía e Informática (INEGI) realizó estudios edafológicos a nivel nacional a tres escalas: 1:1 millón que cubre $100 \%$ del país, 1:250 000 cubre $75 \%$ del país y 1:50 000 cubre 35\% del territorio nacional (INEGI, 1974; 1988a; 1988b).

El Estado de México está cubierto por la cartografía edafológica 1:50 000, de aquí la surge la necesidad de generar una base de datos actualizada. Los sectores involucrados en la planeación demandan información digital actualizada, por municipio, información que es la base en la planeación y ordenamiento de las actividades urbana, agrícola, pecuaria, forestal, minera y pesquera (INEGI, 2015). Por la relevancia y demanda de esta información, en la presente investigación, se plantearon los siguientes objetivos: 1) actualizar la cartografía edafológica 1:50 000 por municipio del Estado de México; y 2) cuantificar la superficie de los suelos y conocer su ubicación para identificar las áreas de reconversión productiva.

\section{Materiales y métodos}

\section{Localización del área de estudio}

El Estado de México se localiza de $18^{\circ} 22^{\prime}$ '14" a $20^{\circ} 17^{\prime} 22^{\prime \prime}$ de latitud norte y de $98^{\circ} 35^{\prime} 35^{\prime \prime}$ a $100^{\circ} 36^{\prime}$ '19" de longitud oeste y tiene una superficie de 2324422 ha. Colinda con los estados de Hidalgo y Querétaro al Norte, Puebla y Tlaxcala al Este, Morelos y Guerrero al Sur y Michoacán al Oeste (INEGI, 2015). Los climas que se presentan en el estado son: templado, semifrío, cálido, semicálido y frío. La temperatura media anual fluctúa de 6 a $28{ }^{\circ} \mathrm{C}$, la precipitación anual va de 600 a 1800 mm, la altura sobre el nivel del mar va de 340 a 5100 m (INEGI, 1999; García, 2004). Los suelos dominantes son: Andosol, Feozem, Vertisol, Regosol y Arenosol (Sotelo et al., 2010). El Estado de México está integrado por 125 municipios (Figura 1), para los cuales se describieron los suelos (INEGI, 2015). 


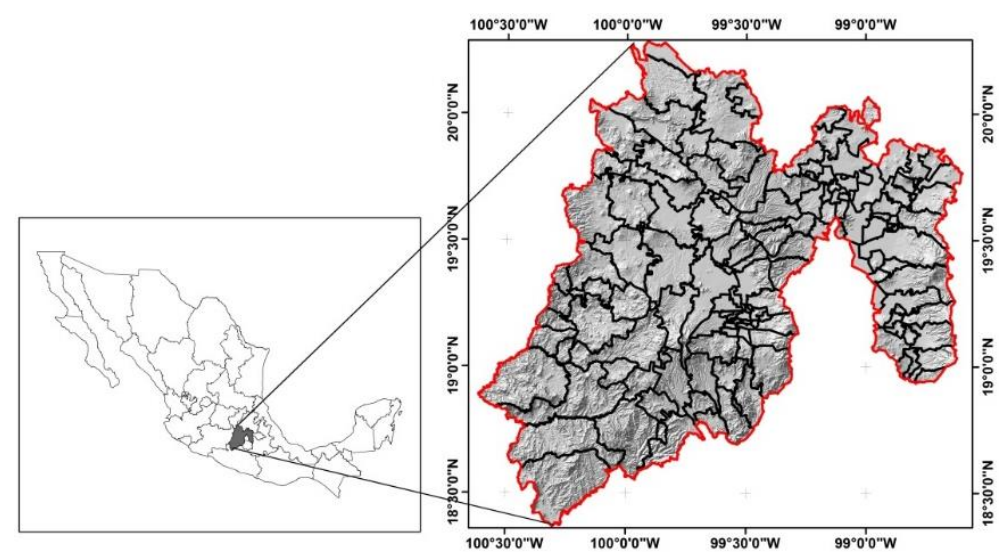

Figura 1. Localización del Estado de México y municipios que lo integran.

\section{Metodología}

Se empleó la información Edafológica del INEGI, escala 1:50 000 (INEGI, 1974). Se digitalizaron 43 cartas que cubren al Estado con Arcinfo versión 6.0 (ESRI, 1992). Las delimitaciones de polígonos y bases de datos generadas fueron: suelos primarios, suelos secundarios, fase física, textura, fase química y profundidad del suelo.

Se realizaron recorridos de campo para verificar y actualizar las Unidades de suelos, mediante barrenaciones y descripción de perfiles de suelos, en los grupos que se tenían dudas con respecto al tipo de suelo presente; los perfiles se ubicaron geográficamente y se muestrearon (FAO, 2009). Las muestras de suelos fueron enviadas al laboratorio para realizar los análisis que utiliza la WRB para clasificar los suelos; estos datos fueron la base para clasificar y actualizar los suelos. La clasificación se realizó con la WRB 2015 (IUSS Working Group WRB, 2015). La actualización de los suelos, su base de datos y la generación de los mapas por municipio se realizó con ArcGIS versión 9.3 (ESRI, 2010).

\section{Resultados y discusión}

El Estado de México presenta 11 unidades de suelos, que son: Andosol, Feozem, Regosol, Vertisol, Cambisol, Leptosol, Luvisol, Acrisol, Solonchak, Fluvisol y Gleysol (Sotelo et al., 2011. En este trabajo, la clasificación de los Planosoles e Histosoles correspondió a los Vertisoles y Feozem respectivamente. Los suelos más productivos del estado son: Feozems, Vertisoles y Cambisoles, por las propiedades físicas y químicas que presentan, como textura media, materia orgánica $>3 \%$, pendiente menor a $10 \%$ y sin fases físicas en la mayoría de los casos. Su superficie se presenta por municipios y se describe la distribución estatal (Figura 2 y Cuadro 1).

\section{Distribución de Feozems}

El Estado de México tiene 472718 ha y representa 20.34\% de la superficie estatal. Los Feozems tienen potencial para la mayoría de los cultivos y especies vegetales, aunque las condiciones climáticas limitan su adaptación y desarrollo. Tienen potencial para especies como maíz, trigo, frijol, papa, zanahoria, cebada, avena, triticale, chícharo, haba, aguacate, durazno, ciruela, 
frambuesa, alfalfa y praderas (SIAP, 2019). Los municipios con mayor superficie son: Aculco, Toluca, Axapusco, Acambay y Jilotepec. Por la superficie que presentan estos suelos, se puede decir que estos municipios tienen el mayor potencial productivo para especies que se adapten a sus condiciones climáticas (Cuadro 1).

a)

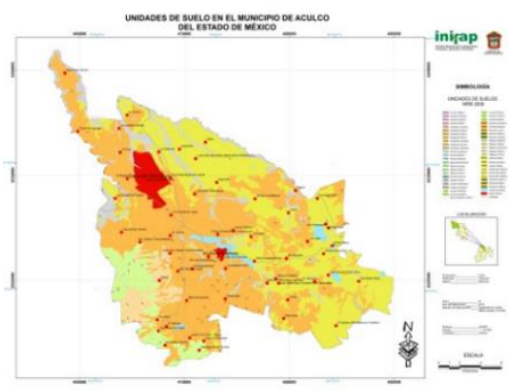

b)

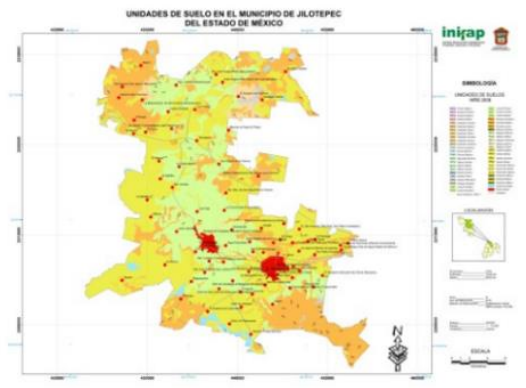

c)

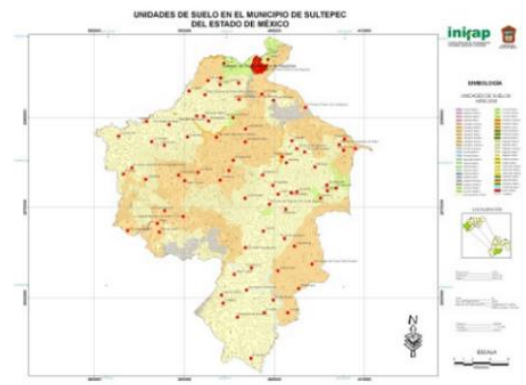

Figura 2. Municipios con mayor superficie de Feozem en a) Aculco, Vertisol; en b) Jilotepec; y Cambisol en c) Sultepec.

Cuadro 1. Municipios con mayor superficie de Feozem, Vertisol y Cambisol.

\begin{tabular}{cccccc}
\hline Municipio & $\begin{array}{c}\text { Feozem } \\
\text { (ha) }\end{array}$ & Municipio & $\begin{array}{c}\text { Vertisol } \\
(\text { ha })\end{array}$ & Municipio & $\begin{array}{c}\text { Cambisol } \\
\text { (ha) }\end{array}$ \\
\hline Aculco & 21654 & Jilotepec & 23808 & Sultepec & 21446 \\
Toluca & 21475 & Almoloya de Juárez & 22587 & Zacualpan & 15889 \\
Axapusco & 20250 & Ixtlahuaca & 20428 & Texcoco & 15123 \\
Acambay & 18856 & Aculco & 15294 & Temascaltepec & 14276 \\
Jilotepec & 15928 & Acambay & 14718 & San Simón de Guerrero & 8964 \\
Hueypoxtla & 15465 & Jocotitlán & 13476 & Amatepec & 8803 \\
Zumpango & 14773 & San Felipe del Progreso & 13467 & Ixtapaluca & 8517 \\
Chapa de Mota & 14613 & Temascalcingo & 10527 & Almoloya de Alquisiras & 6908 \\
Atlacomulco & 12831 & Polotitlán & 10316 & Tejupilco & 6540 \\
Almoloya de Juárez & 12509 & Jiquipilco & 9686 & Texcaltitlán & 6155 \\
Luvianos & 11713 & Tepotzotlán & 8417 & Otzoloapan & 5961 \\
Temascalcingo & 11370 & Atlacomulco & 8227 & Otumba & 5094 \\
\hline
\end{tabular}

\section{Distribución de Vertisoles}

El estado presenta 241485 ha y representan $10.39 \%$ de la superficie del estado. Se localizan en la parte centro y norte del estado. Tienen potencial para especies como maíz, trigo, frijol, triticale, cebada, avena, chícharo, haba, alfalfa y praderas (SIAP, 2019). Los municipios con mayor superficie son: Jilotepec, Almoloya de Juárez, Ixtlahuaca, Aculco, Acambay y Jocotitlán. Estos suelos son muy productivos a nivel estatal, aunque necesitan riego para explotar todo su potencial productivo (Cuadro 1). 


\section{Distribución de Cambisoles}

El estado cuenta con 196047 ha y cubren $8.43 \%$ de la superficie estatal. Son suelos fértiles con muy buen potencial productivo para las especies vegetales que se adapten a las condiciones climáticas de los municipios principalmente para especies como maíz, trigo, frijol, papa, zanahoria, cebada, avena, chícharo, haba, aguacate, durazno, ciruela, frambuesa, alfalfa y praderas (SIAP, 2019). Los municipios con mayor superficie son: Sultepec, Zacualpan, Texcoco, Temascaltepec y San Simón de Guerrero (Cuadro 1).

Los Andosoles, Regosoles y Luvisoles son los que siguen en importancia y superficie. Son menos fértiles, altos contenidos de Al, texturas de medias a gruesa, pendientes $>12 \%$, materia orgánica $<2 \%$ y pH ácido en los tres suelos (Figura 3 y Cuadro 2).

a)

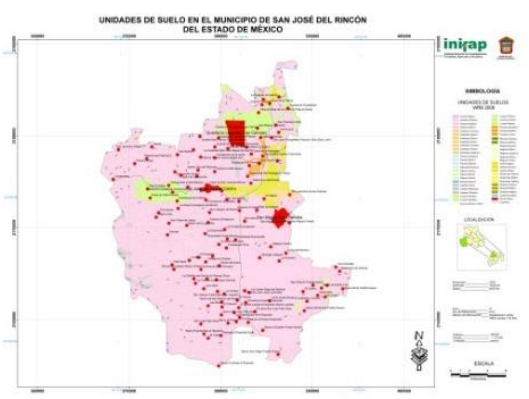

b)

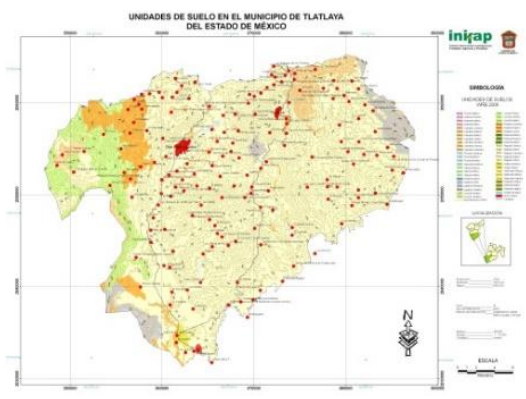

c)

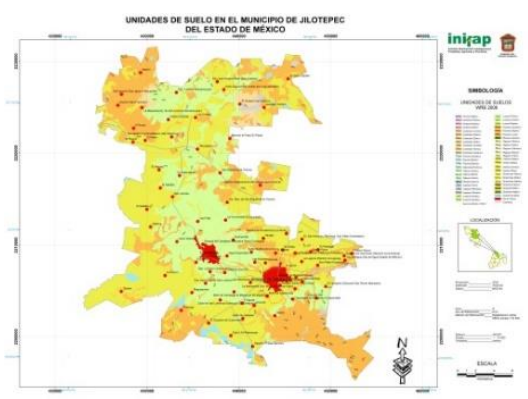

Figura 3. Municipios con mayor superficie de Andosol en a) San José del Rincón, Regosol; en b) Tlatlaya y Luvisol; y en c) Jilotepec.

Cuadro 2. Municipios con mayor superficie de Andosol, Regosol y Luvisol.

\begin{tabular}{cccccc}
\hline Municipio & Andosol (ha) & Municipio & $\begin{array}{c}\text { Regosol } \\
\text { (ha) }\end{array}$ & Municipio & $\begin{array}{c}\text { Luvisol } \\
\text { (ha) }\end{array}$ \\
\hline $\begin{array}{c}\text { San José del Rincón } \\
\text { Villa de Allende }\end{array}$ & 29244 & Tlatlaya & 60889 & Jilotepec & 15853 \\
Ocuilan & 21974 & Amatepec & 41099 & Villa del carbón & 10679 \\
Amanalco & 21616 & Sultepec & 30825 & Acambay & 9876 \\
Temascaltepec & 21314 & Luvianos & 29716 & Almoloya de & 6334 \\
Valle de Bravo & 19885 & Juchitepec & 7396 & Alquisiras & Tlatlaya \\
Zinacantepec & 18867 & Atlautla & 6362 & Morelos & 6056 \\
Villa Victoria & 18031 & Amecameca & 5907 & San Felipe del & 5818 \\
Donato Guerra & 15832 & Zacualpan & 5643 & Coatepec Harinas & 5753 \\
Tenango del Valle & 13668 & Ixtapaluca & 2728 & Jiquipilco & 5565 \\
Coatepec Harinas & 13108 & Tepetlaoxtoc & 2679 & Chapa de Mota & 5181 \\
Tianguistenco & 12610 & Ayapango & 2519 & Otzoloapan & 3799 \\
\hline
\end{tabular}




\section{Distribución de Andosoles}

El Estado de México presenta 479908 ha, cubren la mayor superficie y representa 20.65\% del estado. Se localizan en las partes montañosas del Eje Neovolcánico, Sierra Madre del Sur y en las sierras del Norte del estado. Son suelos de vocación forestal y agrícola. Las especies que tienen potencial productivo en estos suelos son maíz, chícharo, papa, haba, zanahoria, aguacate, durazno, ciruela y frambuesa (SIAP, 2019). Los municipios con mayor superficie son: San José del Rincón, Villa de Allende, Ocuilan, Amanalco, Temascaltepec y Valle de Bravo (Cuadro 2).

\section{Distribución de Regosoles}

El estado presenta 265683 ha y representan el $11.43 \%$ de la superficie estatal. Tienen potencial para especies como maíz, trigo, frijol, papa, cebada, avena, chícharo, haba, aguacate, durazno, ciruela, frambuesa y mango (SIAP, 2019). Los municipios que presentan mayor superficie son: Tlatlaya, Tejupilco, Amatepec, Sultepec y Luvianos. Estos presentan mayor superficie en la parte sur del Estado de México, dónde el clima predominante es tropical (Cuadro 2).

\section{Distribución de Luvisoles}

El Estado de México presenta 146905 ha y cubren 6.32\% de la superficie estatal. Tienen potencial forestal y para frutales como durazno, guayaba, café y aguacate (SIAP, 2019). Los municipios con mayor superficie son: Jilotepec, Nicolás Romero, Villa del Carbón, Acambay y Almoloya de Alquisiras (Cuadro 2).

Los Acrisoles, Fluvisoles y Leptosoles son poco productivos, con vocación forestal, para la producción de hortalizas y para actividades de minería. Los Fluvisoles son suelos poco desarrollados y planos; los Acrisoles son suelos de montaña con textura media y pH ácido, los Leptosoles son suelos delgados 5 a $20 \mathrm{~cm}$, pendiente $>20$ y $<2 \%$ de materia orgánica, su vocación es minera y forestal (Cuadro 3 y Figura 4).

Cuadro 3. Municipios con mayor superficie de Acrisol, Fluvisol y Leptosol.

\begin{tabular}{cccccc}
\hline Municipio & Acrisol (ha) & Municipios & Fluvisol (ha) & Municipio & Leptosol (ha) \\
\hline Luvianos & 11597 & Chalco & 7362 & Zumpahuacán & 14094 \\
Valle de Bravo & 10108 & Amecameca & 5804 & Luvianos & 11797 \\
Villa Victoria & 4385 & Ixtlahuaca & 4972 & Malinalco & 9669 \\
Temascaltepec & 3992 & Tlalmanalco & 1021 & Tepetlaoxtoc & 7623 \\
Ixtapan del Oro & 3931 & Temamatla & 938 & Tejupilco & 7552 \\
Tejupilco & 3671 & Cocotitlán & 892 & Santo Tomás & 5762 \\
Malinalco & 1757 & Jocotitlán & 719 & Otumba & 4753 \\
Donato Guerra & 1704 & Atlautla & 530 & Ocuilan & 4432 \\
El Oro & 1481 & Tenango del Valle & 472 & Tonático & 3978 \\
Villa de Allende & 1318 & Luvianos & 429 & Amatepec & 3887 \\
San Simón de Guerrero & 704 & Zacualpan & 330 & Tlalnepantla de Baz & 3868 \\
Ocuilan & 609 & Morelos & 315 & Temascalcingo & 3823 \\
\hline
\end{tabular}


a)

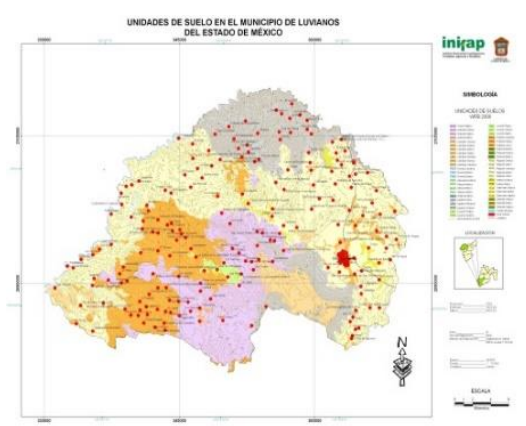

b)

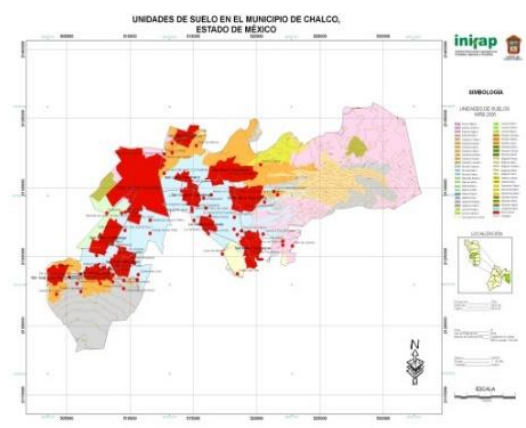

c)

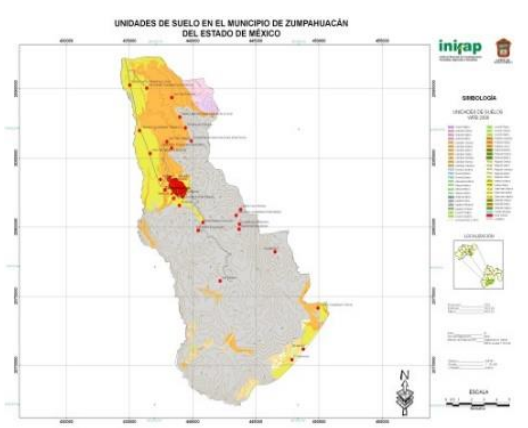

Figura 4. Municipios con mayor superficie de Acrisol en a) Luvianos, Fluvisol; en b) Chalco y Leptosol; y en c) Zumpahuacán.

\section{Distribución de Acrisoles}

El Estado de México presenta 46968 ha y cubre 2.02\% de la superficie estatal. Los Acrisoles son suelos jóvenes, de montaña y baja fertilidad, si se realiza un manejo adecuado de fertilización y conservación, se pueden sembrar algunas especies como aguacate, guayaba, café y durazno (SIAP, 2019). Los municipios con mayor superficie de Acrisoles son: Luvianos, Valle de Bravo, Villa Victoria, Temascaltepec e Ixtapan del Oro (Cuadro 3).

\section{Distribución de Fluvisoles}

El estado presenta 25216 ha y representa $1.08 \%$ del estado. Tienen alta fertilidad natural, porque tienen textura media, son profundos, contenidos de materia orgánica medios, planos y se desarrollan los cultivos que se adapten a las condiciones climáticas presentes; se localizan a las orillas de los ríos, lagos y lagunas. Los municipios con mayor superficie son: Chalco, Amecameca, Ixtlahuaca, Tlalmanalco y Temamatla (Cuadro 3).

\section{Distribución de Leptosoles}

El estado presenta 174968 ha y cubren el $7.53 \%$ de la superficie del estado. El potencial de los Leptosoles es forestal, pecuario, recreativo y minero; las Subunidades Rendzico y Úmbrico tienen potencial para cultivos como maíz, frijol, trigo, cebada, avena y arroz en el estado (SIAP, 2019). Los municipios que presentan mayor superficie son: Zumpahuacán, Luvianos, Malinalco, Tepetlaoxtoc y Tejupilco. En estos suelos, se explota cantera y se extraen otros minerales que demanda la industria minera (Cuadro 3).

Los suelos menos productivos que se presentan en el estado son los Solonchaks y los Gleysoles, por su alta salinidad, sodicidad, texturas gruesas y pendientes irregulares, son suelos con poca superficie y muy localizados, donde sólo crece pasto salado en el caso de los primeros (Cuadro 4 y Figura 5). 
Cuadro 4. Municipios con mayor superficie de Solonchak y Gleysol.

\begin{tabular}{cccc}
\hline Municipio & Solonchak (ha) & Municipio & Gleysol (ha) \\
\hline Texcoco & 7069 & Valle de Chalco Solidaridad & 1345 \\
Nezahualcóyotl & 5937 & Chalco & 542 \\
Ecatepec de Morelos & 5685 & Temascalcingo & 212 \\
Atenco & 5126 & El Oro & 182 \\
Tecámac & 2878 & Chapa de Mota & 166 \\
Chimalhuacán & 2585 & Teoloyucan & 95 \\
Valle de Chalco Solidaridad & 2554 & Zumpango & 84 \\
Zumpango & 1856 & Jilotepec & 57 \\
Nextlalpan & 1679 & & \\
Tultitlán & 773 & & \\
La paz & 753 & & \\
Tezoyuca & 445 & & \\
\hline
\end{tabular}

a)

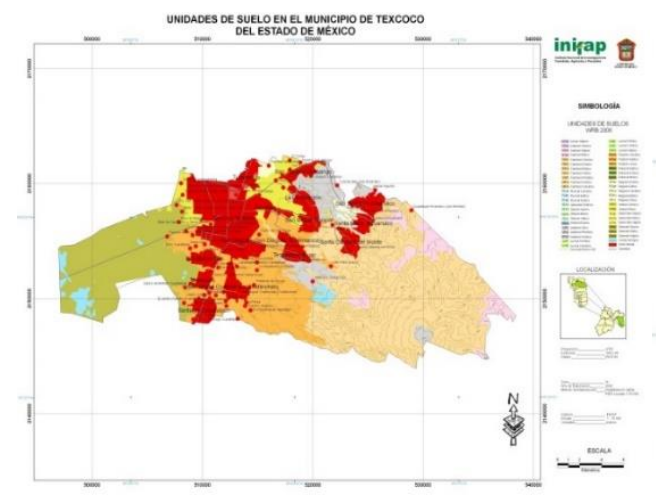

b)

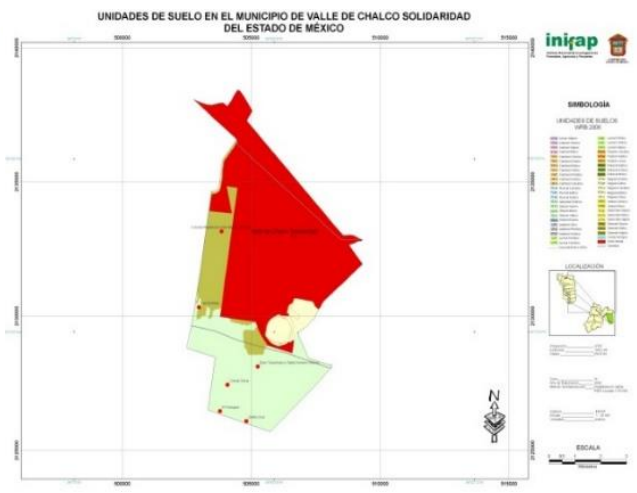

Figura 5. Municipios con mayor superficie de Solonchak en a) Texcoco y Gleysol; y en b) Valle de Chalco Solidaridad.

\section{Distribución de Solonchaks}

El Estado de México está cubierto por 39290 ha y representa 1.69\% de la superficie estatal. Estos suelos presentan características de salinidad, conductividad eléctrica y $\mathrm{pH}$ altos, por lo que es muy difícil el desarrollo de cultivos; el potencial es para pasto salado y para la explotación de sal en las partes donde la salinidad es muy alta. Los municipios con estos suelos son: Texcoco, Nezahualcóyotl, Ecatepec de Morelos y Atenco (Cuadro 4).

\section{Distribución de Gleysoles}

La superficie en el Estado de México es de 2765 ha y solo representa $0.12 \%$ de los suelos del estado. Tienen una fertilidad natural baja y su vocación es forestal; sólo se puede sembrar algunas especies con un manejo adecuado de fertilización. Los principales municipios con Gleysoles son: Valle de Chalco Solidaridad, Chalco, Temascalcingo y El Oro (Cuadro 4). 
La información de suelos es necesaria, porque los datos son cruciales en la planeación del uso del suelo, el manejo, estudios ambientales, estudios de erosión, conservación y estudios con modelos, que simulan el crecimiento de los cultivos y calculan anticipadamente el rendimiento (Nachtergaele et al., 2000; Adhikari et al., 2014; Jafari et al., 2014). Existen muy pocos estudios de suelos y las escalas de representación son entre 5 y 25 millones. La FAO tiene estudios de suelos a nivel continental con escalas de 1:25 millones, 1:15 millones y 1:5 millones (FAO, 1993; FAO, 1996; FAO, 2008).

Los estudios de suelos en los Estados Unidos de América son escala 1:7.5 a 1:15 millones (Soil Survey Staff, 1998). En este sentido, Brevik y Hartemink (2013) usan la taxonomía y determinan que los suelos dominantes en USA son: Molisol, Alfisol, Entisol, Inceptisol, Aridisol, Ultisol y Vertisol; además, Lytle (2000); USDA (2000); VanEngelen (2000) realizaron estudios de suelos y diseñaron una base de datos (NASIS), la cual facilitó la adquisición, el manejo y la ubicación de los suelos.

A nivel internacional, se han realizado estudios similares a los de esta investigación, como los del centro internacional de información referencial de suelos (CIIRS), dónde se diseñó una base de datos con la información de suelos del mundo para dar a conocer los estudios de suelos, que se realizan en los diferentes países (ISRIC, 2000). Por su parte, Adhikari et al. (2014) elaboran un mapa nacional de suelos de Dinamarca, el cual se basó en la leyenda de la FAO-UNESCO, usando técnicas de mapeo digital de suelos, con observaciones de perfiles de suelos y datos ambientales. Esta información es la base para los estudios de planeación, manejo, conservación y evaluación del impacto ambiental del país.

En el caso de México, están los estudios de suelos del INEGI con las escalas 1:1 000 000, 1:250 000 y 1:50 000 (INEGI, 1970; INEGI, 1974) al respecto, el INEGI no cuenta con cartografía digital de ninguna escala; por ello, los resultados de este trabajo son muy importantes, ya que presentan la cartografía digital escala 1:50 000, dónde los suelos dominantes son los Andosol, Feozem, Vertisol, Regosol y Cambisol. Por su parte, la Secretaría del Medio Ambiente y Recursos Naturales (SEMARNAT) establece las bases para la realización y actualización de los estudios de suelos en México (SEMARNAT, 1999).

A nivel nacional se reportan 18 unidades (INEGI, 1974), de las cuales 11 se presentan en el todo el Estado de México, siendo los suelos dominantes: Andosol, Feozem, Regosol, Vertisol y Cambisol que son también los más productivos. Sotelo et al. (2010); Sotelo et al. (2011) realizaron la actualización de los suelos del Estado de México a escala 1:50 000, a nivel de Distrito de Desarrollo Rural (DDR), destacando que el suelo es uno de los principales recursos naturales, en el cual se sustentan los bosques y la producción de alimentos. Además, indican que contar con información actualizada sobre el tipo de suelo y su distribución facilita la planeación y clasificación de las actividades agropecuarias. Brevik y Hartemink (2013) mencionan, que en Estados Unidos de América los mapas de suelos se empezaron a genera a principios del siglo veinte, como una necesidad de conocer el manejo y conservación de este recurso.

Por los suelos presentes en los municipios del Estado de México, se recomienda que se seleccione el cultivo o la especie a establecer, tomando en cuenta las condiciones climáticas y la finalidad de la producción, si es para autoconsumo o comercial. No se recomienda establecer zonas urbanas en municipios que presentan suelos fértiles y con poca pendiente. Existen tipos de suelos que tienen 
una vocación para explotación minera y desarrollo urbano, como los Leptosoles. Los desarrollos urbanos en los municipios deben de implementarse en estos suelos y prohibir la construcción de fraccionamientos en suelos con vocación agrícola como: los Feozem, Cambisoles, Vertisoles y Andosoles.

Finalmente, la información generada es una herramienta fundamental en la planeación de las actividades agropecuarias de los municipios del Estado de México, por el detalle de la información, la cual está a nivel de municipio. Los municipios del Estado de México, con este estudio, saben los tipos de suelos que tienen y la superficie que abarcan, por lo tanto, conocen si la vocación de sus suelos es: agrícola, pecuaria, forestal, urbana o minera y consecuentemente pueden implementar mejores planes de desarrollo municipal.

\section{Conclusiones}

Los suelos presentes con mayor superficie en el Estado de México son: Andosol (20.65\%), Feozem (20.34\%), Regosol (11.43\%), Vertisol (10.39\%), Cambisol (8.43\%), Leptosol (7.53\%), Luvisol (6.32\%), Acrisol (2.02\%), Solonchak (1.69\%), Fluvisol (1.08\%) y Gleysol (0.12\%). Los mejores suelos para la agricultura y producción de alimentos a nivel mundial por sus propiedades físicas y químicas y que dominan en el Estado de México son: Feozems con 472718 ha, Vertisoles con 241 485, Cambisoles con 196047 y Andosoles con 479908 ha. Los Feozem se presentan en Aculco, Toluca, Axapusco, Acambay y Jilotepec, mientras que los Vertisoles destacan en Jilotepec, Almoloya de Juárez, Ixtlahuaca, Aculco y Acambay.

Los Cambisoles presentan mayor superficie en los municipios de Sultepec, Zacualpan, Texcoco, Temascaltepec y San Simón de Guerrero, mientras que los Andosoles sobresalen en San José del Rincón, Villa de Allende, Ocuilan, Amanalco y Temascaltepec. Los municipios del Estado de México que tienen excelentes suelos, para la producción de cultivos anuales y frutales de ciclo perenne dependiendo del clima presente, son Aculco, Toluca, Jilotepec, Almoloya de Juárez, Ixtlahuaca, Sultepec, Zacualpan, Texcoco, Villa de Allende y Temascaltepec.

\section{Literatura citada}

Adhikari, K.; Minasny, B.; Greve, M. B. and Greve, M. H. 2014. Constructing a soil class map of Denmark based on the FAO legend using digital techniques. Geoderma. 214-215:101-113.

Behrens, T. and Scholten, T. 2006. Digital soil mapping in Germany-a review. J. Plant Nutr. Soil Sci. 169(3):434-443.

Behrens, T.; Schmidt, K.; A. X. Zhu, A. X. and Scholten, T. 2010a. The ConMap approach for terrain-based digital soil mapping. Eur. J. Soil Sci. 61(1):133-143.

Behrens, T.; Zhu, A.; Schmidt, K. and Scholten, T. 2010b. Multi-scale digital terrain analysis and feature selection for digital soil mapping. Geoderma. 155(3-4):175-185.

Bockheim, J. G. and Gennadiyev, A. N. 2000. The role of soil-forming processes in the definition of taxa in Soil Taxonomy and the World Soil Reference Base. Geoderma. 95(1-2):53-72.

Brady, N.C. and Weil, R. R. 1999. The nature and properties of soils. Prentice Hall. Twelfth Edition. Upper Saddle River, New Jersey, United States of America. 881 p.

Brevik, E. C. 2009. The teaching of soil science in geology, geography, environmental science and agricultural programs. Soil Survey Horizon. 50(4):120-123. 
Brevik, E. C. 2010. Collier cobb and allen d. hole: geologic mentors to early soil scientists. Physics and Chemistry of the Earth. 35(15-18):887-894.

Brevik, E. C. and Hartemink, A. E. 2010. Early soil knowledge and the birth and development of soil science. Catena. 83(1):23-33.

Brevik, E. C. and Hartemink, A. E. 2013. Soil Maps of the United States of America. Soil Sci. Soc. Am. J. 77(4):1117-1132.

ESRI. 1992. Environmental Systems Research Institute. Arc info. New York, USA. 125 p.

ESRI. 2010. Environmental Systems Research Institute. ArcGis 9.3. New York, USA. 286 p.

FAO. 1988. Food and Agriculture Organization of the United Nations. Soil Map of the World. Revised Legend. World Soil Resources Report 60. FAO. Rome, Italy. 136 p.

FAO. 1993. Food and Agriculture Organization of the United Nations. World soil resources. An explanatory note on the FAO World Soil Resources Map at 1:25,000,000 scale. World Soil Resources Report No. 66, Rev. 1. FAO. Rome, Italy. 268 p.

FAO. 1996. Food and Agriculture Organization of the United Nations. The digitized soil map of the world including derived soil properties. CD-ROM. FAO. Rome, Italy. 527 p.

FAO. 2008. Food and Agriculture Organization of the United Nations. Nueva base de datos mundial sobre el suelo. Roma, Italia. http://www.fao.org/newsroom/ES/news/2008/ 1000882/index.html.

FAO. 2009. Food and Agriculture Organization of the United Nations. Guía para la descripción de suelos. Cuarta Edición. Rome, Italy. 99 p.

FAO-ISRIC y SICS. 1999. Organización de las Naciones Unidas para la Agricultura y la Alimentación- Centro Internacional de Referencia e Información en Suelos y Sociedad Internacional de las Ciencias del Suelo. Base Referencial Mundial del Recurso Suelo. FAO. Roma, Italia. $93 \mathrm{p}$.

FAO-UNESCO. 1970. Food and Agriculture Organization of the United Nations/United Nations Educational, Cientific and Cultural Organization. Soil Map of the World 1:1 000000 . Vol. I. Legend. Paris, France. 59 p.

García, E. 2004. Modificaciones al sistema climático de Köppen para la República Mexicana. 5a Ed. Instituto de Geografía. Serie de libros No. 6. Universidad Nacional Autónoma de México (UNAM). México, DF. 292 p.

Hengl, T. and Rossieter, G. D. 2003. Supervised landform to enhance and replace photointerpretation in semi-detailed soil survey. Soil Science Society of American Journal. 67(6):1810-1822.

INEGI. 1970. Instituto Nacional de Estadística, Geografía e Informática. Cartografía de México Escala 1:250 000. Secretaría de Programación y Presupuesto. México, D. F.

INEGI. 1974. Instituto Nacional de Estadística, Geografía e Informática. Cartografía Escala 1:50 000. Secretaría de Programación y Presupuesto. México, D. F.

INEGI. 1988a.Instituto Nacional de Estadística, Geografía e Informática. Atlas Nacional del medio físico. 1a reimpresión. Secretaría de Programación y Presupuesto. México, D. F. 235 p.

INEGI. 1988b. Instituto Nacional de Estadística, Geografía e Informática. Síntesis Geográfica. Nomenclatura y Anexo Cartográfico del Estado de México. Secretaría de Programación y Presupuesto. México, D. F. 635 p.

INEGI. 1999. Instituto Nacional de Estadística, Geografía e Informática. Anuario Estadístico del Estado de México. Aguascalientes, Ags., México. 596 p.

INEGI. 2015. Instituto Nacional de Estadística, Geografía e Informática. Estadísticas básicas del Estado de México. Síntesis Geográfica del Estado de México. http://www.inegi.gob.mx. 
ISRIC. 2000. International soil reference and information centre. GLASOD, SOTER, Other soil databases International Soil Reference and Information. Centre Wageningen, The Netherlands. www.isric.nl.

IUSS Grupo de Trabajo. WRB. 2007. International unión of soil science grupo de trabajo base mundial de suelos. 2007. Base referencial mundial del recurso suelo. Primera actualización informes sobre recursos mundiales de suelos No. 103. FAO. Roma, Italy. 117 p.

IUSS Working Group. WRB. 2006. International union of soil science - international soil reference and information Centre and Food and Agriculture Organization of the United Nations. (IUSS- ISRIC and FAO). World reference base for soil resources. A framework for international classification, correlation and communication. $2^{\text {nd }}($ Ed.). World Soil Resources Reports No. 103. Rome, Italy. 128 p.

IUSS Working Group. WRB. 2015. International union of soil science. World reference base for soil resources 2014, update 2015. International soil classification system for naming soils and creating legends for soil maps. World Soil Resources Reports No. 106. FAO. Rome, Italy. 192 p.

Jafari, A.; Khademi, H.; Finke, P. A.; Van de Wauw, J. and Ayoubi, S. 2014. Spatial prediction of soil great groups by boosted regression trees using a limited point dataset in an arid region, southeastern Iran. Geoderma. 232-234:148-163.

Kozlova, N. D. and Konyushkova, V. M. 2009. State of the art and prospects of digital soil mapping: the results of the third global workshop (Logan, USA, 2008). Eurasian Soil Science. 42(6):699-702.

Lytle, D. J. 2000. United States soil survey database. In: hand book of soil science. Sumner, M. E. (Ed.). New York, USA. H53-H67 pp.

McBratney, B. A.; Mendonca-Santos, L. M. and Minasny, B. 2003. On digital soil mapping. Geoderma. 117(1-2):3-52.

Nachtergaele, F. O.; Spaargaren, O.; Deckers, J. A. and Ahrens, B. 2000. New developments in soil classification world reference base for soil resources. Gerodermia. 96(4):345-357.

Ortiz, S. C.; Pájaro, H. D. y Gutiérrez, C. M. C. 1994. Introducción a la leyenda del mapa mundial de suelos FAO/UNESCO, versión 1988. Programa de Edafología. Instituto de Recursos Naturales. Colegio de Postgraduados. Montecillo, Texcoco, Estado de México. 40 p.

Porta, C. J.; López, A. R. M. y Roquero, D. C. 2003. Edafología. Para la agricultura y el medio ambiente. $3^{\text {a }}$ (Ed.). Ediciones Mundi-Prensa. México, DF. 929 p.

Rosa, A. S.; Heuvelink, G. B. M.; Vasques, G. M. and Anjos, L. H. C. 2015. Do more detailed environmental covariates deliver more accurate soil maps?. Geoderma. 243-244:214-227.

SEMARNAP. 1999. Secretaría de Medio Ambiente, Recursos Naturales y Pesca. Mapas de suelos dominantes de los Estados Unidos Mexicanos. SEMARNAP. México, DF. 22 p.

Shi, X.; Long, R.; Dekett, R. and Philippe, J. 2009. Integrating different types of knowledge for digital soil mapping. Soil Sci. Soc. Am. J. 73(5):1682-1692.

SIAP. 2019. Servicio de Información Agroalimentaria y Pesquera. Cierre de la producción agrícola 2019 por municipios para el Estado de México. http://www.siap.gob.mx/cierre-de-laproduccion-agricola-por-estado/.

Soil Survey Staff. 1960. Soil conservation service. Soil classification. A comprehensive system. $7^{\text {th }}$ Approximation. United State Department of Agriculture (USDA). Washington, DC. USA. 265 p.

Soil Survey Staff. 1998. Soil taxonomy. A basic system of soil classification for marking and interpreting soil surveys. $2^{\text {nd }}$ (Ed.). United State Department of Agriculture (USDA). Natural Resources Conservation Service (NRCS). Washington, DC. USA. 326 p. 
Soil Survey Staff. 2014. Keys to soil taxonomy. $12^{\text {th }}$ (Ed.). United State Department of Agriculture (USDA). Natural Resources Conservation Service (NRCS). Washington, DC. USA. 360 p.

Sotelo, R. E. D.; González, H. A.; Cruz, B. G.; Moreno, S. F. y Cruz, C. G. 2011. Los suelos del Estado de México y su actualización a la base referencial mundial del recurso suelo. Re. Mex. Cienc. Fores. 2(8):71-84.

Sotelo, R. E. D.; González, H. A.; Cruz, B. G.; Moreno, S. F. y Ochoa, E. S. 2010. La clasificación FAO-WRB y los suelos del Estado de México. INIFAP. Zinacantepec, Estado de México. $159 \mathrm{p}$.

Spaargaren, C. O. 2000. Other systems of soil classification. In: hand book of soil science. Sumner, M. E. (Ed.). New York, USA. E137-E174 pp.

USDA. 2000. United States Department of Agriculture. World Soil Resources Natural Resources Conservation Service. United States Department of Agriculture. Washington, DC. www.nhq.usda.gov/WSR/.

Wilding, L. P. 2000. Classification of soil. In: hand book of soil science. Sumner, M. E. (Ed.). New York, USA. E175-E392 pp.

Zhu, X. A.; Hudson, B.; Burt, J.; Lubich, K. and Simonson, D. 2001. Soil mapping using GIS, expert knowledge, and fuzzy logic. Soil Sci. Soc. Am. J. 65(5):1463-1472. 